\title{
Modelling the Ionospheric Signatures of Geomagnetic Pulsations
}

\author{
P. R. SutClifFe \\ Hermanus Magnetic Observatory, P. O. Box 32, Hermanus 7200, South Africa
}

(Received November 30, 1993; Revised April 1, 1994; Accepted May 5, 1994)

\begin{abstract}
Oscillating phase shifts are frequently observed in ionospherically propagated HF radio waves in association with ULF geomagnetic pulsations. These Ionospheric Signatures of Pulsations (ISP) are observed in ionospherically reflected radio waves transmitted from ground stations, in radio signals transmitted from satellites to ground stations, and in the backscattered signals received by HF over-the-horizon radars. We review the techniques used to model the relationship between ISPs and geomagnetic pulsations. Model results for a variety of geophysical conditions are compared with observations.
\end{abstract}

\section{Introduction}

A variety of ionospheric signatures are observed in association with ULF geomagnetic pulsations. In this review we are mainly concerned with those signatures which manifest themselves as oscillating frequency shifts in $\mathrm{HF}$ radio waves propagating through the ionosphere. These ionospheric signatures of pulsations (ISPs) have been most frequently observed as oscillating Doppler frequency shifts of HF radio waves reflected in the ionosphere. More recently, oscillations in amplitude and group range of ionospherically reflected radio waves have also been observed.

Figure 1 is a schematic representation of the ionospheric process. As a radio wave enters a region containing electrons whose concentration increases with height, the phase velocity increases due to the decrease of the phase refractive index and the wave is refracted or, for vertical incidence, reflected. The presence of a ULF geomagnetic pulsation will cause the refractive index to be modulated at the pulsation frequency, thus causing characteristics of a reflected radio wave also to be modulated.

We commence this review with a brief presentation of some examples of the ionospheric signatures of ULF geomagnetic pulsations. We then develop a model which demonstrates some

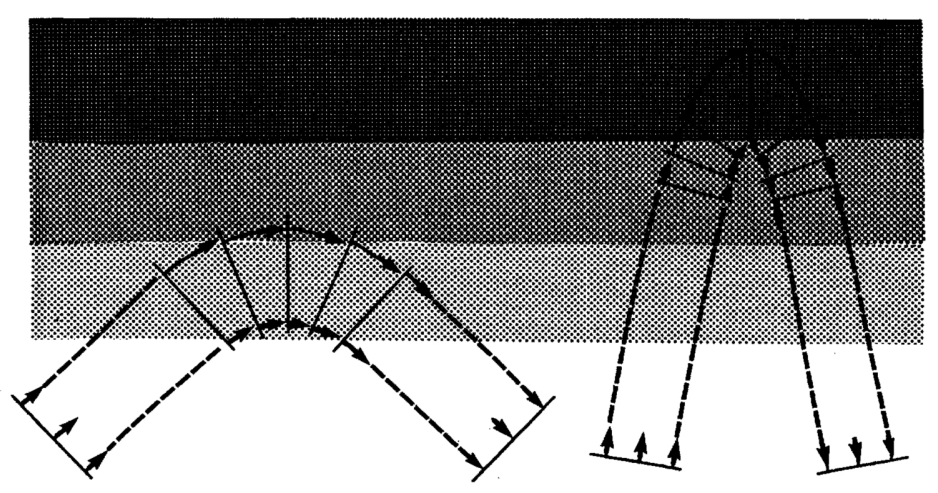

Fig. 1. Schematic diagram illustrating the refraction and reflection of radio waves in the ionosphere. 
of the mechanisms connecting these signatures to geomagnetic pulsations. Finally, we evaluate the model by comparing computed predictions with observations.

\section{Examples of Observations}

Three decades ago a number of researchers reported observations of a correlation between rapid geomagnetic variations and frequency shifts in HF radio waves received obliquely after ionospheric reflection from distant stations (Chan et al., 1962; Duffus and Boyd, 1968). Subsequently there have been numerous reports dealing specifically with observations of ground-based ULF geomagnetic pulsations and simultaneous Doppler frequency shifts in HF radio waves reflected by the ionosphere at vertical incidence (Klostermeyer and Röttger, 1976; Menk et al., 1983; Sutcliffe and Poole, 1984; Watermann, 1987; Kolokolov and Marakhovskiy, 1988; Jarvis and Gough, 1988; Tedd et al., 1989; Yumoto et al., 1989; Menk, 1992). An example of Doppler frequency oscillations in association with $\mathrm{Pi} 2$ pulsations is shown in Fig. 2. An example of ionospheric oscillations, expressed in terms of Doppler velocity, in association with Pc3 pulsations is shown in Fig. 3.

There have been observations of a related phenomenon, namely, a correlation between ULF geomagnetic pulsations and oscillations in carrier phase of radio signals from a geostationary satellite received at a ground station (Davies and Hartmann, 1976; Okuzawa and Davies, 1981). These oscillations have been ascribed to oscillations in the total electron content (TEC) along the radio ray path and have consequently been termed TEC pulsations. An example of ATS-6 40 $\mathrm{MHz}$ carrier phase oscillations recorded at Boulder is shown in Fig. 4. Comparisons indicate that there is generally good agreement of TEC pulsations with simultaneous geomagnetic pulsations on the ground; however, there is a lack of or poor agreement with geomagnetic pulsations at geostationary orbit (Okuzawa and Davies, 1981). This suggests that TEC pulsations originate mainly in the ionosphere.

Rapid Doppler frequency variations related to geomagnetic pulsations have also been reported in the backscattered signals received by over-the-horizon radars (Bourdillon et al., 1989). An

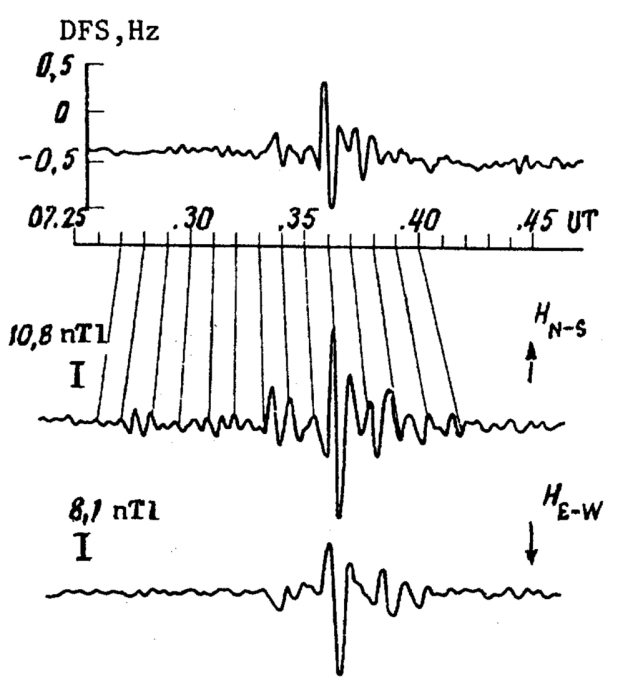

Fig. 2. Examples of synchronous oscillations of the Doppler Frequency Shift of a radio signal reflected from the ionospheric F-region during vertical sounding and the intensities of the horizontal components of the geomagnetic field recorded on September 23, 1985, at Petropavlovsk-Kamchatskiy (after Kolokolov and Marakhovskiy, 1988). 

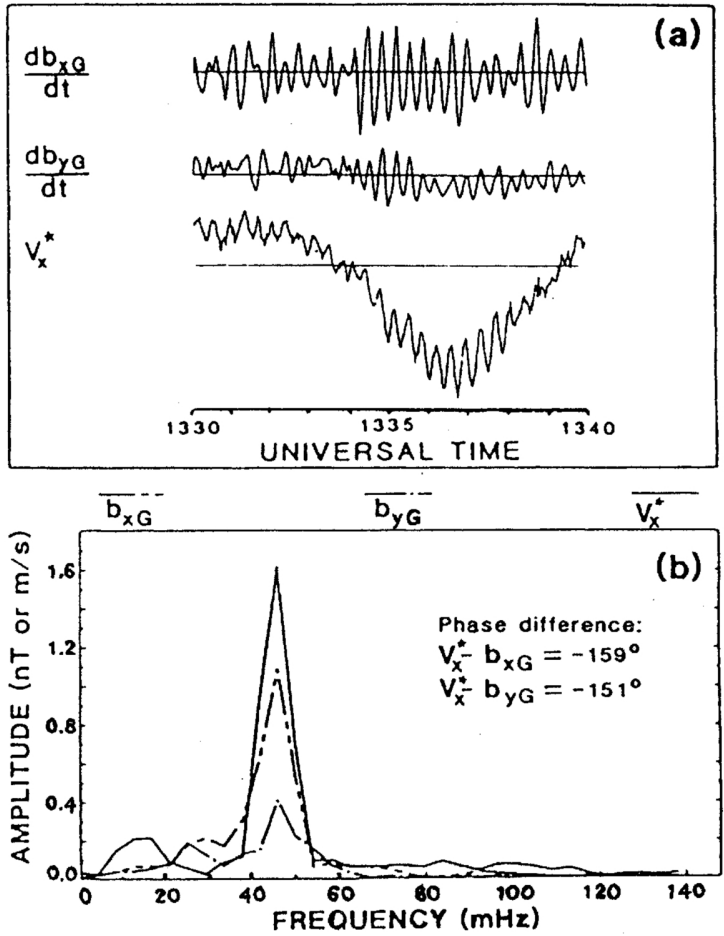

Fig. 3. (a) Example of Pc3 pulsations and Doppler velocity oscillations recorded at Grahamstown on 30 November 1983. (b) Spectra for the 4 minute segment of pulsations commencing at 1334 UT (after Sutcliffe and Poole, 1984).

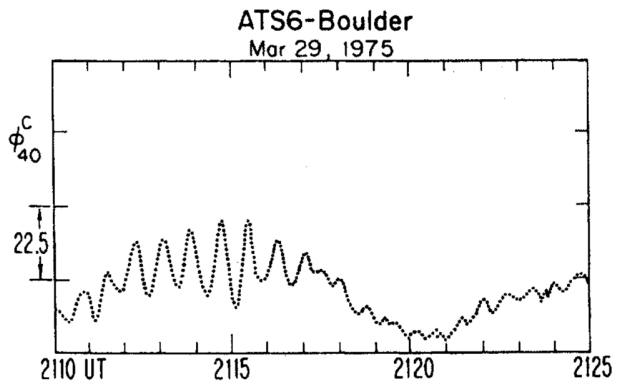

Fig. 4. Computer output at Boulder on March 29, 1975, showing oscillations in the ATS-6 $40 \mathrm{MHz}$ carrier phase with periods near $50 \mathrm{sec}$ (after Davies and Hartmann, 1976).

example of Doppler shift oscillations related to Pc3 pulsations is shown in Fig. 5. Bourdillon et al. (1989) suggest that, provided signal processing is adapted, a backscatter radar should be able to provide information not previously accessible on the structure of geomagnetic pulsation fields at ionospheric heights, especially at mid-latitudes.

Recently observations have been made of oscillations of other ionospheric parameters in association with ULF geomagnetic pulsations. Aslin et al. (1991) reported the first observations 

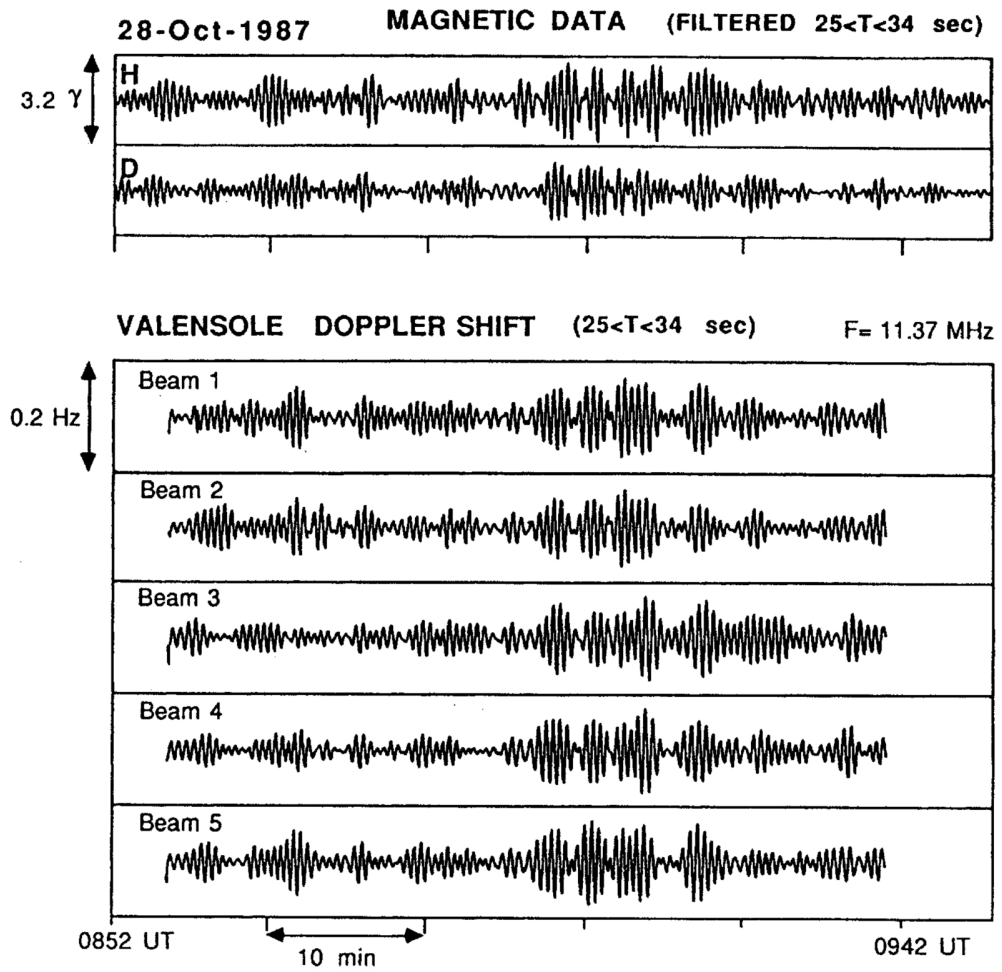

Fig. 5. Pc3 data recorded on October 28, 1987 and filtered in the band $25<T<34$ sec. The two upper curves show the ground magnetic components $\mathrm{H}$ and $\mathrm{D}$ recorded at Auray. The five lower curves show the Doppler shift variations in the five radar azimuths at frequency $f=11.37 \mathrm{MHz}$ (after Bourdillon et al., 1989).

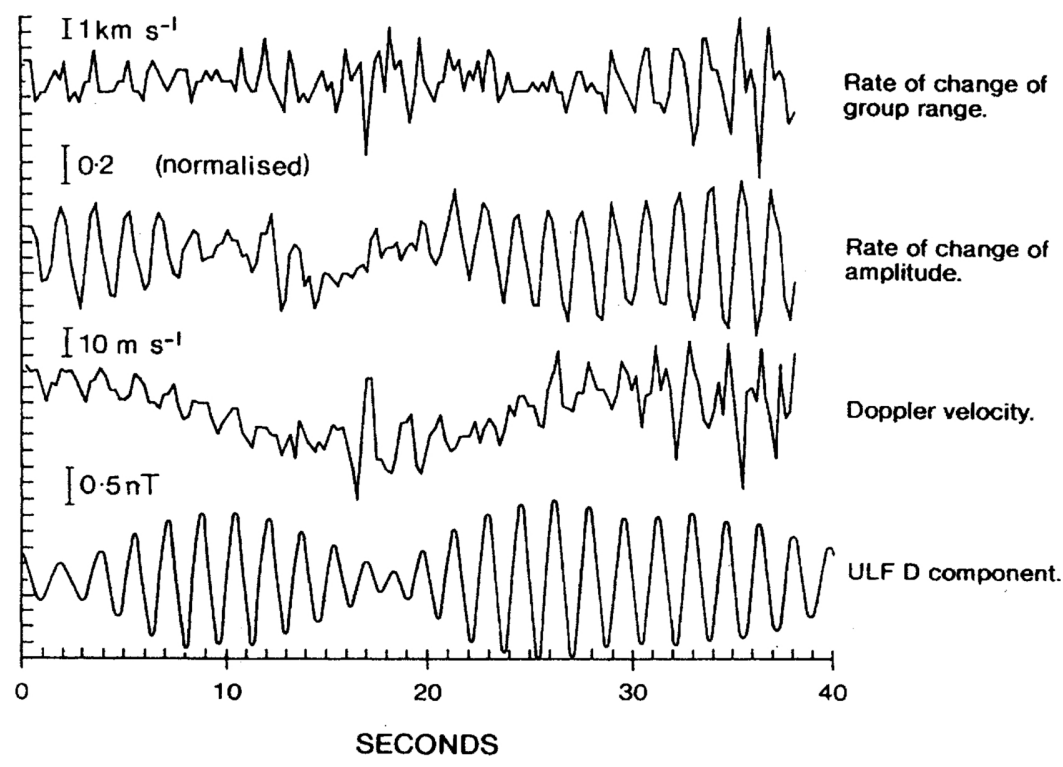

Fig. 6. Example of ionospheric group range, echo amplitude, and Doppler velocity oscillations in association with a Pc1 geomagnetic pulsation (after Aslin et al., 1991). 
of the ionospheric signatures of Pc1 pulsations in ionosonde echo data. Besides oscillations in Doppler velocity, they observed oscillations of echo amplitude, group range, and echo location. An example of ionospheric oscillations in association with Pc1 pulsations is shown in Fig. 6.

\section{Derivation of a Model}

Rishbeth and Garriott (1964) were the first to develop a theory to account for the ionospheric oscillations associated with geomagnetic pulsations. They proposed two mechanisms to explain the association of Doppler velocity oscillations with geomagnetic variations. In their "dynamomotor" theory, an alternating $\mathbf{E}$ field was assumed to be generated in the E-region ionosphere by dynamo action. The geomagnetic oscillations observed on the ground were assumed to be caused by the current driven by this ionospheric $\mathbf{E}$ field. The Doppler velocity oscillations were taken to be due to the vertical component of the bulk motion of the ionosphere caused by the $\mathbf{E} \times \mathbf{B}$ drift of plasma associated with the oscillating $\mathbf{E}$ field. In their "hydromagnetic" approach, Rishbeth and Garriott (1964) assumed a hydromagnetic wave travelling along geomagnetic field lines to cause sinusoidal distortions of the field and plasma. They associated the vertical component of these plasma oscillations with the horizontal component of the field oscillations and assumed further that the magnetic field oscillations at ground level are equal to those in the ionosphere. Rishbeth and Garriott (1964) described their theoretical models as crude, and pointed out certain shortcomings. In an attempt to improve on this work, Jacobs and Watanabe (1966) derived an expression for the change in value of the refractive index due to a change in the distribution of the ionospheric electrons. However, in their derivation they regarded as insignificant a term which Poole et al. (1988) showed to be important. In addition, none of these authors was able to take account of the effect of the atmosphere and ionosphere on the incident hydromagnetic wave, as has been subsequently described by Hughes and Southwood (1976). A concise review of these ISP models and their shortcomings has been given by Sutcliffe and Poole (1989).

Over the years most researchers have attempted to couch their observations in terms of the above-mentioned theories. However, in a more recent detailed study Watermann (1987) found that these theoretical models were not adequately supported by the observations and consequently required major revisions. Poole et al. (1988) developed a significantly improved model for the vertical incidence case in which they showed that at least three distinct mechanisms could account for the observed relationship. Although developed to account specifically for Doppler velocity oscillations in the case of vertical incidence, this model is also able to explain the presence of TEC pulsations (Poole and Sutcliffe, 1987). Poole et al.'s (1988) model was able to eliminate some of the failings of the earlier models, but still suffered from shortcomings; some of these shortcomings were addressed in a further improvement by Sutcliffe and Poole (1989). The model derivation which we review here is essentially that of Sutcliffe and Poole (1989).

For a radio wave vertically incident upon the ionosphere the phase height is given by:

$$
h^{*}=\int_{0}^{z_{R}} \mu d z
$$

where integration with respect to $z$ is in the vertical direction, $\mu$ is the real part of the refractive index given by the Appleton-Hartree formula, and $z_{R}$ is the height of reflection. The Doppler velocity $V^{*}$, defined as the time rate of change of the phase height for the reflected radio wave, is given by:

$$
V^{*}=\frac{d h^{*}}{d t}=\frac{d}{d t} \int_{0}^{z_{R}} \mu d z
$$

The use of the term "velocity" derives from conventional radar where the Doppler frequency shift of the returning echo is directly related to the velocity of the solid target. However, as 
will be seen, changes in phase height of reflection in the ionosphere need not necessarily imply movement of the reflection level, and for this reason "velocity" is perhaps an unfortunate usage. In many research papers the term Doppler frequency shift $\Delta f$ is used. Its relationship to $V^{*}$ is given by:

$$
\Delta f=2 f_{R} V^{*} / c
$$

where $f_{R}$ is the radio sounding frequency and $c$ is the speed of light. However, we have described our theory in terms of the Doppler velocity $V^{*}$ rather than the Doppler frequency because the former is independent of the sounding frequency $f_{R}$.

In the derivation of an expression for the Doppler velocity $V^{*}$, we assume that the refractive index $\mu$ varies with oscillations in both the electron concentration $N$ and the geomagnetic induction $\mathbf{B}=\mathbf{B}_{0}+\mathbf{b} e^{-i \omega t}$ where $\mathbf{B}_{0}$ is the background field and $\mathbf{b}$ and $\omega$ are the pulsation field amplitude and angular frequency, respectively. Explicitly expressing this functional dependence, the Doppler velocity in Eq. (2) can be written:

$$
V^{*}=\frac{d}{d t} \int_{0}^{z_{R}} \mu\left[B_{L}(z, t), B_{T}(z, t), N(z, t)\right] d z
$$

where $B_{L}$ and $B_{T}$ are the components of the Earth's magnetic induction parallel (longitudinal) and transverse to the radio wave direction respectively. Carrying out the time differentiation yields:

$$
V^{*}=\int_{0}^{z_{R}}\left[\frac{\partial \mu}{\partial B_{L}} \frac{\partial B_{L}}{\partial t}+\frac{\partial \mu}{\partial B_{T}} \frac{\partial B_{T}}{\partial t}+\frac{\partial \mu}{\partial N} \frac{\partial N}{\partial t}\right] d z
$$

We now make use of the continuity equation for electrons in the ionosphere in which it has been assumed that production and loss terms can be neglected, thus:

$$
\frac{\partial N}{\partial t}=-\nabla N \cdot \mathbf{v}-N(\nabla \cdot \mathbf{v})
$$

where $\mathbf{v}$ is the electron drift velocity. Combining Eqs. (5) and (6) we can express $V^{*}$ in terms of three separate mechanisms, that is:

$$
V^{*}=V_{1}+V_{2}+V_{3}
$$

where

$$
\begin{gathered}
V_{1} \approx-i \omega \int_{0}^{z_{R}}\left[\frac{\partial \mu}{\partial B_{L}} b_{z}+\frac{\partial \mu}{\partial B_{T}} b_{x}\right] d z \\
V_{2}=-\int_{0}^{z_{R}}\left[\frac{\partial \mu}{\partial N} \frac{\partial N}{\partial z} v_{z}\right] d z
\end{gathered}
$$

and

$$
V_{3}=-\int_{0}^{z_{R}}\left[\frac{\partial \mu}{\partial N} N(\nabla \cdot \mathbf{v})\right] d z
$$

The "magnetic" mechanism 1, i.e. $V_{1}$, arises from changes in the refractive index through its dependence on the magnetic field intensity. The "advection" mechanism 2, i.e. $V_{2}$, arises from changes in the ionospheric refractive index due to the vertical component of the bulk motion of electrons in the ionosphere. The "compression" mechanism 3, i.e. $V_{3}$, arises from changes in the refractive index due to the divergence of electron velocity along the ray path. Note that if production and loss terms, which we assumed to be zero in Eq. (6), are not negligible, then a fourth mechanism will arise. The latter would likely be the situation if the model were to be applied to observations at auroral latitudes. Since the prime objective of this work has been 
to apply the model to the many sets of observations which have been made at low- and midlatitudes, it is reasonable to assume that there are no oscillatory production and loss mechanisms of electrons operating in the ionosphere (Poole and Sutcliffe, 1987).

The above equations have been used to compute the Doppler velocity $V^{*}$ for a great variety of conditions. In all cases it has been found that $V_{1}$ is one to two orders of magnitude less than either $V_{2}$ or $V_{3}$; this is illustrated in the next section on model predictions. Consequently, it would be reasonable to ignore the magnetic mechanism, as was done in the earlier model by Jacobs and Watanabe (1966).

The Doppler velocity predicted by earlier models and which was ascribed to the vertical motion of the reflection level (Rishbeth and Garriott, 1964), is essentially an approximation of the advection mechanism $V_{2}$. The way in which $V_{2}$ can be identified with the vertical motion of the reflection level can be seen by making the following coarse approximations to the expression for $V_{2}$. Assume that the electron concentration $N$ is horizontally uniform and that the refractive index $\mu$ is independent of $\mathbf{B}$, then:

$$
\frac{d \mu}{d z} \cong \frac{\partial \mu}{\partial N} \frac{\partial N}{\partial z}
$$

Further assume that the vertical component of electron velocity $v_{z}$ is independent of height, consistent with the idealised picture of the whole ionosphere moving up and down uniformly. With these assumptions the expression for $V_{2}$ becomes:

$$
\begin{aligned}
V_{2} & =-\int_{0}^{z_{R}}\left[\frac{\partial \mu}{\partial N} \frac{\partial N}{\partial z} v_{z}\right] d z \\
& =-\int_{0}^{z_{R}}\left[\frac{\partial \mu}{\partial z} v_{z}\right] d z \\
& =-v_{z} \int_{0}^{z_{R}} d \mu \\
& =v_{z} .
\end{aligned}
$$

In some of the past literature there has been the tendency to equate Doppler velocity $V^{*}$ to the vertical component of bulk electron velocity $v_{z}$ at the reflection level. The above analysis shows that this assumption would be approximately true if $V_{2}$ given by Eq. (9) were the only significant contributor to $V^{*}$, particularly if $v_{z}$ were constant with height. In Fig. 7 we compare the amplitudes and phases of $v_{z}$ with $V_{2}$ and $V^{*}$, each computed using the same set of geophysical parameters. It is clear that although there are similarities between $v_{z}$ and $V_{2}$, especially in the phase, $v_{z}$ and $V^{*}$ are markedly different; this difference arises mainly from the major contribution of $V_{3}$ to $V^{*}$. We thus point out that attempts to associate the Doppler velocity with the vertical velocity of the reflection level will not generally be valid.

The compression mechanism $V_{3}$ is also related to the effect of electron concentration on the refractive index, but due to more localized variations. Two main contributions can be identified here. The first and major contribution, which was shown to be important by Poole et al. (1988), arises from the divergence of the transverse component of electron velocity and is associated with field line compression. This can be demonstrated by making simplifying assumptions to the full expression for $V_{3}$ given by Eq. (10). Assume that the drift velocity of ionization is due to the oscillating ionospheric electric field $\mathbf{E}$ and given by:

$$
\mathbf{v} \cong \mathbf{E} \times \mathbf{B} / B^{2}
$$

where $\mathbf{B}$ is the geomagnetic induction as previously defined. This assumption implies that the effect of collisions on electrons is ignored and that the field-aligned component of electron velocity 

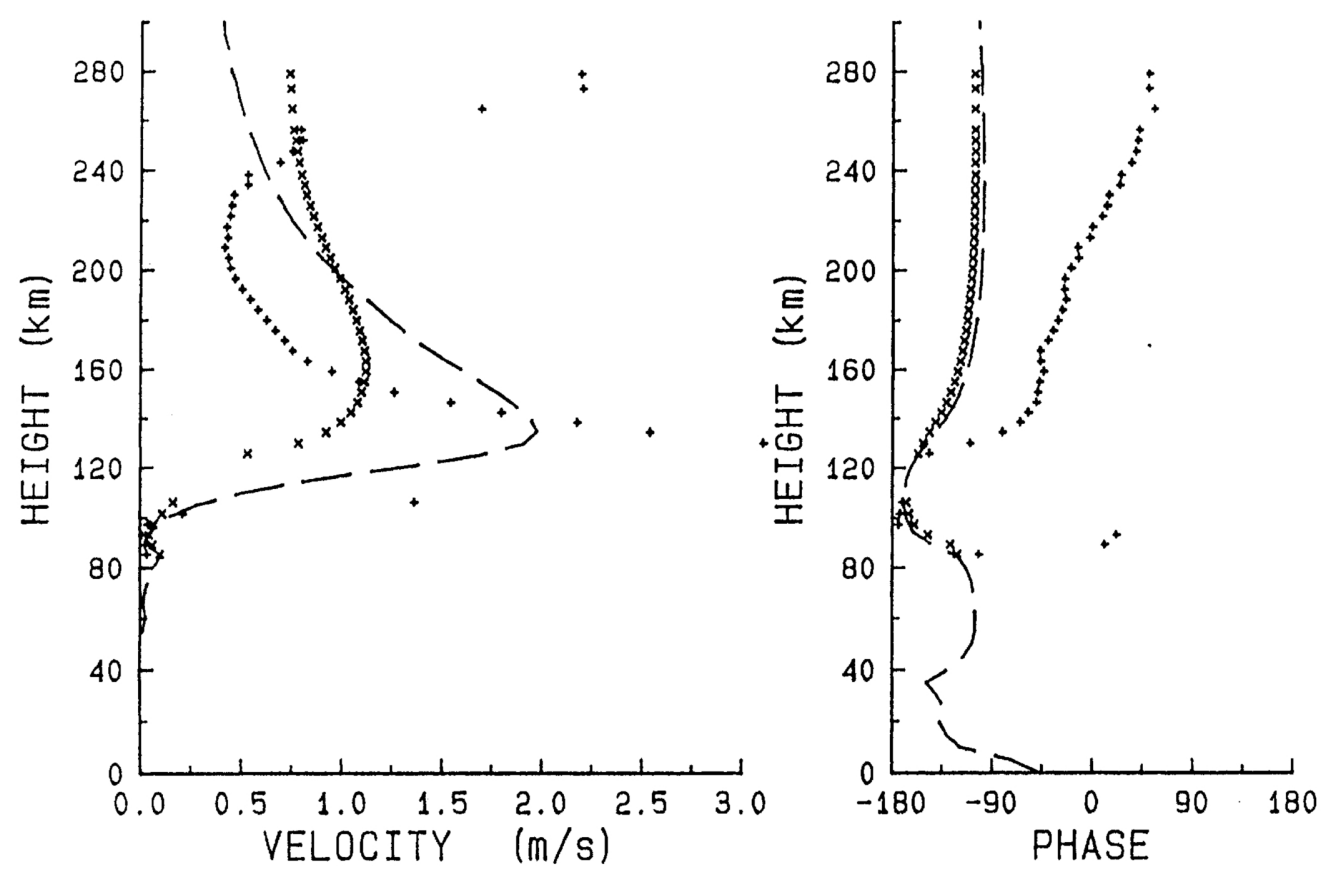

Fig. 7. Comparison of (a) the amplitudes and (b) the phases of $v_{z}$ (long-dashed line) with $V_{2}(\times)$ and $V^{*}(+)$ (after Sutcliffe and Poole, 1989).

is zero. The divergence term in Eq. (10) becomes:

$$
\begin{aligned}
\nabla \cdot \mathbf{v} & =\mathbf{B} \cdot \nabla \times \mathbf{E} / B^{2}-\mathbf{E} \cdot(\nabla \times \mathbf{B}) / B^{2}+(\mathbf{E} \times \mathbf{B}) \cdot \nabla\left(B^{-2}\right) \\
& \simeq \mathbf{B} \cdot\left(-\frac{\partial \mathbf{b}}{\partial t}\right) / B^{2} \\
& =i \omega b_{p} / B
\end{aligned}
$$

where $b_{p}$ is the field-aligned component of the pulsation field and numerical evaluation shows that at ULF frequencies the 2nd and 3rd terms are 2 to 4 orders of magnitude less than the 1st term. Thus:

$$
\begin{aligned}
V_{3} & =-\int_{0}^{z_{R}}\left[\frac{\partial \mu}{\partial N} N(\nabla \cdot \mathbf{v})\right] d z \\
& \simeq-i \omega \int_{0}^{z_{R}}\left[\frac{\partial \mu}{\partial N} \frac{N b_{p}}{B}\right] d z .
\end{aligned}
$$

The second contribution to $V_{3}$, which was first reported by Sutcliffe and Poole (1989), arises from the divergence of the field-aligned component of electron velocity. They demonstrated that the commonly held assumption that field-aligned or vertical currents are zero is not a good one, and that such currents contribute significantly to the electron velocity vector and its divergence. This in turn has a pronounced effect on $V^{*}$ through Eqs. (9) and (10). Collisions are taken into account in the calculations for both of these contributions.

The quantitative determination of $V^{*}$ requires the numerical computation of Eqs. (8), (9), and (10). The following briefly summarizes the steps necessary to achieve this. A detailed description of the procedure is given by Poole et al. (1988). 
i) Compute the direct, Pedersen, and Hall conductivities and electron mobilities as functions of height through the ionosphere, for an observed or model electron concentration profile.

ii) Compute the pulsation electric and magnetic field profiles through the ionosphere using, for example, the method of Hughes and Southwood (1976) or Fujita (1987).

iii) Utilise the electron mobility and electric field profiles computed above to compute $v_{z}$ and $\nabla \cdot \mathbf{v}$ as functions of height through the ionosphere. In the computation of $\nabla \cdot \mathbf{v}$, values of $v_{z}(z)$ from adjacent height steps are used to determine $\partial v_{z} / \partial z$, while the horizontal wave numbers $k_{x}$ and $k_{y}$ are used in the determination of $\partial v_{x} / \partial x$ and $\partial v_{y} / \partial y$.

iv) Numerically integrate the expressions for $V_{1}, V_{2}$, and $V_{3}$ in Eqs. (8), (9), and (10). For this purpose, waves for both magnetoionic modes can be assumed to propagate vertically up from the ground to the reflection level applicable to the radio frequency under consideration. Finally, the contributions $V_{1}, V_{2}$, and $V_{3}$ are added, taking account of their relative phases, to give the total Doppler velocity $V^{*}$.

The expressions presented thus far were derived to explain the relationship between ionospheric Doppler velocity oscillations and geomagnetic pulsations. Recent observations of oscillations in other ionospheric parameters such as ionosonde echo amplitude and group range in association with Pc1 pulsations (Aslin et al., 1991), require extensions to these expressions. We previously derived an expression for the Doppler velocity from:

$$
V^{*}=\frac{d}{d t} \int_{0}^{z_{R}} \mu d z
$$

where $\mu$ is the real part of the ionospheric phase refractive index. Expressions for other ionospheric signatures can be derived from similar expressions and in a similar manner. For example, the time rate of change of absorption of a radio wave is given by (Sutcliffe, 1992):

$$
\dot{A}=\frac{d}{d t} \frac{4 \pi f_{R}}{c} \int_{0}^{z_{R}} \chi d z
$$

where $\chi$ is the imaginary part of the phase refractive index. Similarly, the time rate of change of group range is given by

$$
\dot{h}^{\prime}=\frac{d}{d t} \int_{0}^{z_{R}} \mu^{\prime} d z
$$

where $\mu^{\prime}$ is the ionospheric group refractive index.

\section{Model Predictions}

The above model, developed by Poole et al. (1988) and Sutcliffe and Poole (1989), can be utilized to predict the Doppler velocities expected for specified geophysical conditions. Sutcliffe and Poole (1990) made a detailed study of the relationship between Doppler velocity oscillations and geomagnetic pulsations by using the model. They demonstrated how variable the relationship between $V^{*}$ and the components of $\mathbf{b}_{g}$ (where the subscript $g$ refers to $\mathbf{b}$ at ground level) can be, both in amplitude and phase, depending on the prevailing set of geophysical parameters. Parameters which were varied in their study included radio sounding frequency, electron concentration profile, magnetoionic mode, geomagnetic inclination and pulsation frequency and wave number. In this section we consider some general aspects of the model predictions. In the next section we consider certain particular aspects of the predictions and how they relate to observations.

For any set of geophysical parameters, the value of $V^{*}$ will also depend on an experimental variable, namely the radio sounding frequency. The variation of $V^{*}$ with sounding frequency is in turn intimately related to the shape of the electron concentration profile. Thus, in order to assess $V^{*}$ for a particular set of geophysical parameters, it is convenient to plot height profiles 
calculated for stepwise increments in radio sounding frequency, in which each calculated value of $V^{*}$ is plotted at the real height at which reflection of that frequency would take place. We show examples of such profiles for $V^{*}$ and its component parts $V_{1}, V_{2}$, and $V_{3}$ in Figs. 8 and 9 for the O-mode, calculated for a typical set of geophysical parameters. The electron concentration profiles used were taken from the International Reference Ionosphere (IRI 90), and are typical for a mid-latitude station (Hermanus $34.4^{\circ} \mathrm{S}, 19.2^{\circ} \mathrm{E}$ ) for ionospheric conditions during summer and sunspot maximum at local midday and midnight respectively. Other typical parameters used in the computations were inclination $I=-60^{\circ}$, background field intensity $B_{0}=30000 \mathrm{nT}$, pulsation period $T=2 \pi / \omega=20 \mathrm{~s}$, NS scale length $L_{x}=2 \pi / k_{x}=500 \mathrm{~km}$, and EW scale length $L_{y}=2 \pi / k_{y}=6000 \mathrm{~km}$. Note that the results are normalized to give $b_{x}$ (NS pulsation field) at ground level an amplitude of $1 \mathrm{nT}$ and phase of $0^{\circ}$.

We briefly consider the significance of some of the results. In Fig. 8 the absence of values for $V^{*}$ in the height interval between 102 and $120 \mathrm{~km}$ is due to a valley between the E- and F-regions in the electron concentration profile used for the computations, that is, the region from which no reflections are obtained. The profiles in Figs. 8 and 9 show that the amplitude of $V_{1}$ is small, although not insignificant, compared with $V_{2}$ and $V_{3}$ for all reflection heights in the ionosphere. The amplitude of $V_{3}$ tends to be greater than that of $V_{2}$ during the day-time; however, at night $V_{2}$ is significantly greater. The phases of $V_{2}$ and $V_{3}$ are such that the amplitude of $V^{*}$ is less than the amplitudes of $V_{2}$ or $V_{3}$ in much of the F-region. The Doppler velocity attains local maxima just above the upper limits of the ionization valley between the E- and F-regions and near the F-region peak. This behaviour is due to a similar behaviour in $V_{3}$ which increases rapidly when the electron concentration gradient approaches zero and group retardation is greatest; however, reflections from these regions are unlikely to be observed in practice. A noticeable feature of the Doppler velocity amplitudes predicted in Figs. 8 and 9 is that they are approximately an order of magnitude greater in the F-region than in the E-region.

In order to establish the dependence of Doppler velocity oscillations on variations of the electron concentration profile, Sutcliffe and Poole (1990) compared results computed using International Reference Ionosphere profiles for various local times, seasons, and sunspot numbers. The following summarises some of their findings:

i) on average, $V^{*}$ tends to increase slightly with decreasing sunspot number;

ii) values of $V^{*}$ vary very little with season;

iii) $V_{2}$ increases on average towards winter;

iv) $V_{3}$ decreases on average towards winter;

v) in most cases, the change in phase with reflection height in the F-region tends to be gradual for a particular set of geophysical parameters, but highly variable between different sets of parameters.

We conclude this section by mentioning, without discussion, a number of other general model predictions reported by Sutcliffe and Poole (1990). The differences in $V^{*}$ for O- and X-mode reflections are very small and arise from the anti-phase relationship of the $V_{1}$ components for the two modes. Doppler velocity amplitude is a function of inclination and shows a general trend to initially decrease and then increase with increasing inclination. Doppler velocity amplitudes differ significantly, depending whether the pulsation field phase motion is poleward or equatorward. In summary it may be stated that the relationship between $V^{*}$ and the components of $\mathbf{b}_{g}$ depends on many parameters and can show extreme variability according to the prevailing ionospheric and magnetospheric conditions. The relationship depends most significantly on the shape of the electron concentration profile. 


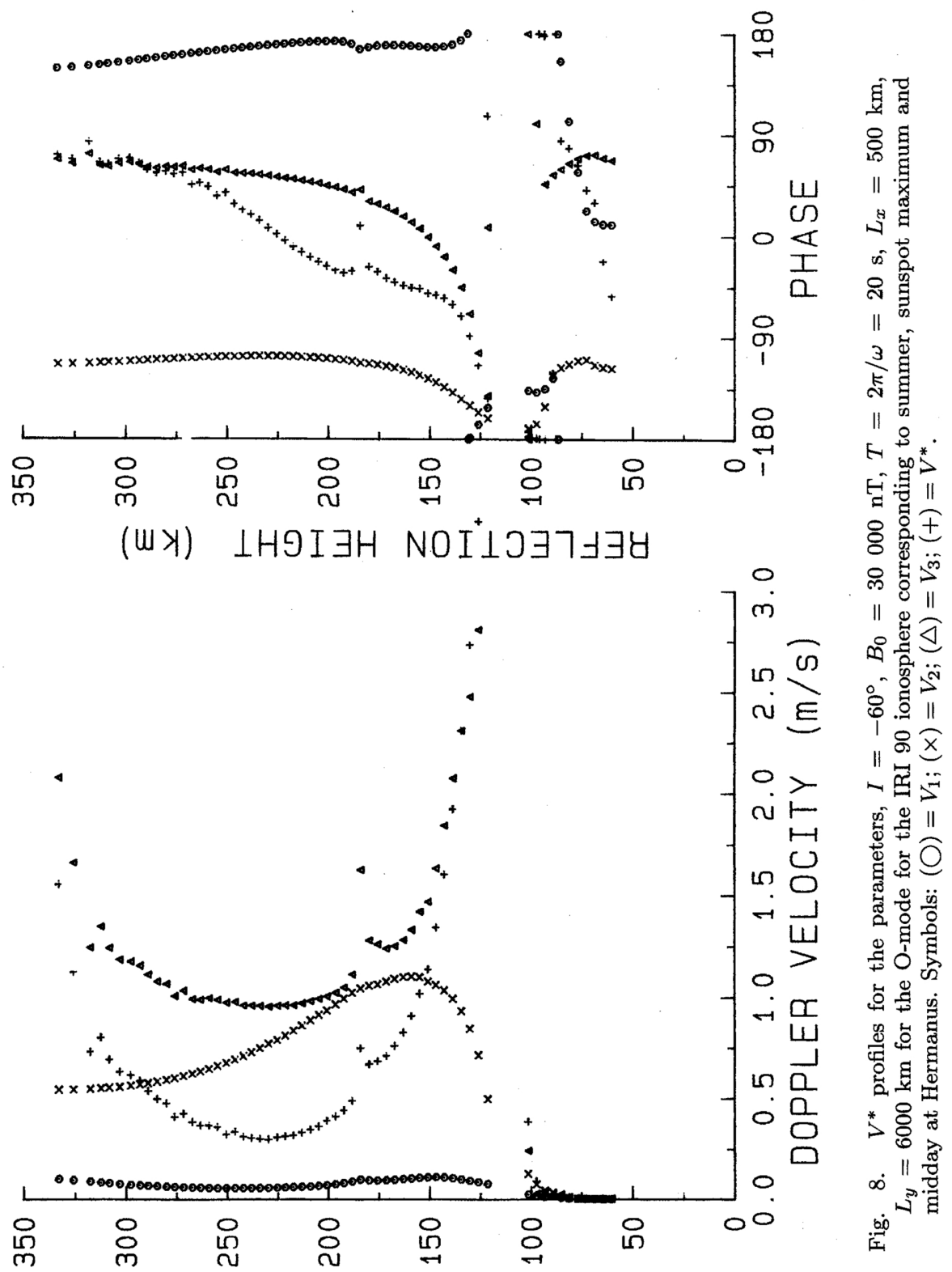

(ய> 


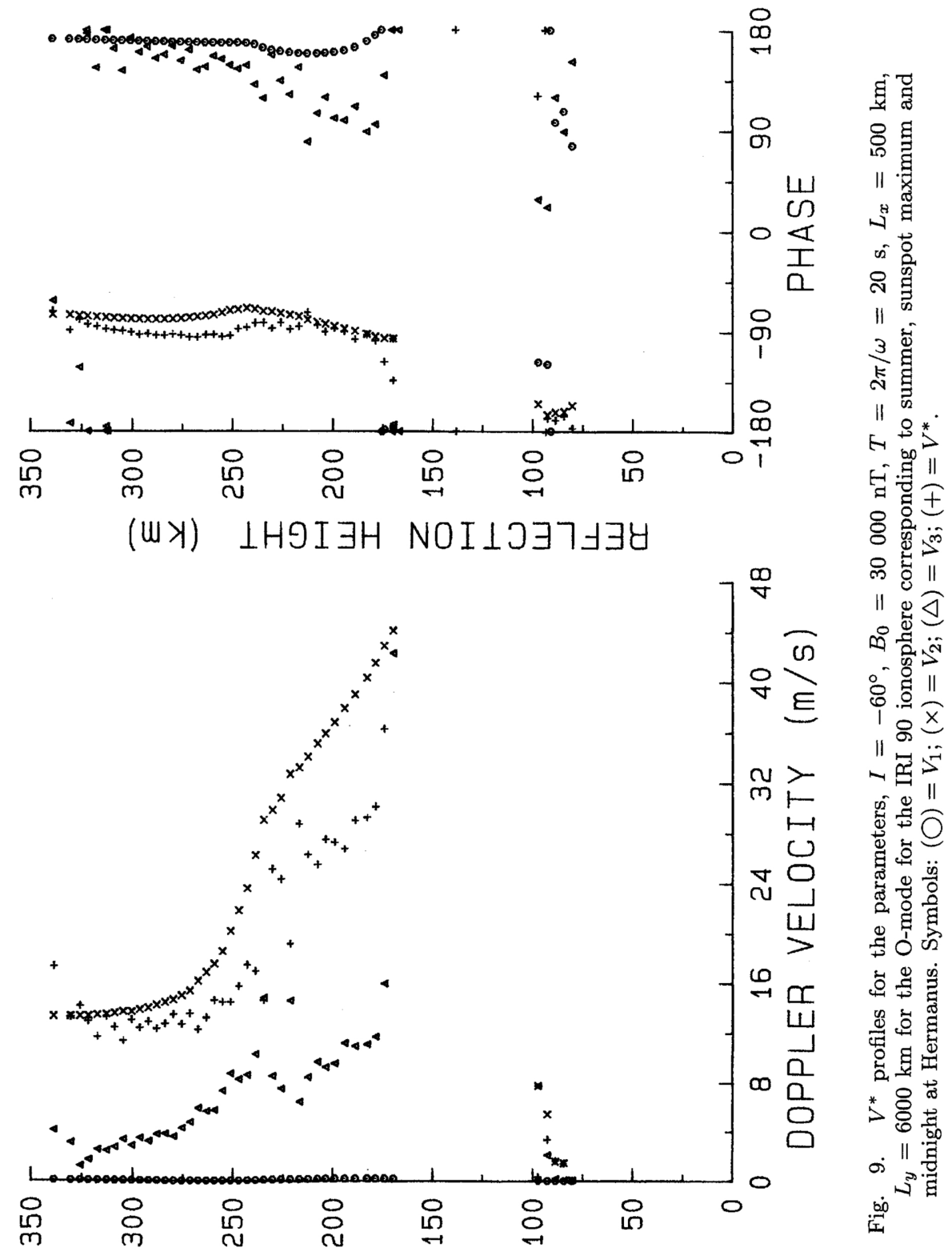

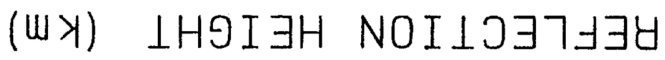




\section{Comparison of Model Predictions with Observations}

The prime motivation for developing the model which we have reviewed above, was that previous models did not agree sufficiently with observations. Consequently, it is essential to test the validity and shortcomings of the present model. In order to do this, we compare the relationships between Doppler velocity oscillations and geomagnetic pulsations predicted by the model (Sutcliffe and Poole, 1990) with those reported in the literature.

\subsection{Agreement of model predictions with observations}

We commence our comparison by considering the amplitude ratio of Doppler velocity oscillations to geomagnetic pulsations. Model predictions of the amplitude ratio for parameters typical of long period pulsations vary from a few tenths of a meter to some tens of meters per second per nanoTesla (Sutcliffe and Poole, 1990). This is illustrated in Figs. 8 and 9 where the Doppler velocity amplitudes were computed for a pulsation with an $\mathrm{N}-\mathrm{S}$ component of $1 \mathrm{nT}$ at ground level. These amplitudes are in good agreement with the results of a detailed statistical study of observations of SIs and Pi2s presented by Watermann (1987); the mean amplitude ratios plotted in his Fig. 4 vary in the range from 0.3 to $20 \mathrm{~ms}^{-1} \mathrm{nT}^{-1}$. The amplitude ratios observed by Jarvis and Gough (1988) and Menk (1992) in association with Pc3 and Pi2 pulsations also fall within this range.

A noticeable feature of the Doppler velocity amplitudes predicted by the model and again illustrated in Figs. 8 and 9 is that they are approximately an order of magnitude greater in the F-region than in the E-region. A limited set of observations made during 1983 by Sutcliffe and Poole (1985) strongly supports this prediction. Observational evidence for smaller Doppler velocity amplitudes from E-region reflections associated with Pc3-4 pulsations is also provided by the results of Jarvis and Gough (1988) and Tedd et al. (1989); the latter stated that the poorer correlation between the E-region Doppler data and the magnetic variations is a common observation. Al'Perovich et al. (1989) made observations of $V^{*}$ oscillations in association with Pi2 pulsations at a number of radio sounding frequencies. They also found that the oscillations from E-region reflections are barely observable for these night-time pulsations. In all the examples presented by Sutcliffe and Poole (1990), it is clear that the amplitude of Doppler velocity oscillations is significantly enhanced near the ionospheric cusps; this is also in accord with observations (Menk, 1992).

The model predicts an increase in Doppler velocity to geomagnetic pulsation amplitude ratio at night compared to day-time conditions. Figures 8 and 9 show the $V^{*}$ profiles for the same station, O-mode, summer and sunspot maximum, but for midday and midnight respectively. A comparison shows that the amplitudes of $V^{*}$ are 10-30 times greater at night than during the day, depending on reflection height. This increase is due mainly to a similar increase in $V_{2}$; the amplitude of $V_{1}$ remains almost unchanged while $V_{3}$ is $2-10$ times greater at night. The reason for this behaviour is to be found in the amplitude relationship between the day- and night-time electric and magnetic fields. Because of lower E-region Pedersen and Hall conductivities at night, a larger night-time electric field is required to produce E-region currents sufficient to sustain the magnetic field components measured on the ground. Al'Perovich et al. (1989) reported that the amplitude ratios predicted by the model were 5-10 times lower than for their night-time observations of $\mathrm{Pi} 2$ pulsations. However, they erroneously compared their observations with the predictions of Poole et al. (1988) for day-time conditions. When the correct night-time conditions are applied to the model, the predictions are in fact very close to the observational results of Al'Perovich et al. (1989) for Pi2 pulsations. Bourdillon et al.'s (1989) observations of Pc3 and Pi2 pulsations and Watermann's (1987) comparison of day- and night-time SSCs and SIs also lend support to the model prediction of a greater $V^{*}$ to $b_{x}$ amplitude ratio at night. In contrast, however, the observations of Menk (1992) do not exhibit an increase in amplitude ratio for night-time Pi2 
pulsations.

Sutcliffe and Poole (1990) showed that the amplitude of Doppler velocity oscillations is a function of pulsation frequency and wave number. The $V^{*}$ amplitude has a tendency to increase with oscillation frequency. The detailed behaviour, however, is more complex and frequency dependent, but agrees reasonably well with Watermann's (1987) statistical analysis of observations. Aslin et al. (1991) extrapolated the model results of Poole et al. (1988) to Pc1 frequencies and found that the predicted amplitudes were in good agreement with the amplitudes of Doppler velocity oscillations observed in association with Pc1 pulsations. According to Menk (1992), the Doppler velocity oscillations associated with Pc3-4 pulsations increase in amplitude with increasing frequency, while night-time $\mathrm{Pi} 2$ events show no such relationship. The model predicts a very rapid increase of Doppler velocity amplitude with increasing wave number (Sutcliffe and Poole, 1990). This dependence is similar to that exhibited by the attenuation of the horizontal magnetic and electric fields between the magnetosphere and the ground due to evanescent decay in the atmosphere (Hughes and Southwood, 1976). This is probably not unexpected, since $V_{1}, V_{2}$, and $V_{3}$ each depend on one or other component of $\mathbf{E}$ or $\mathbf{b}$. Menk (1992) found that when the most probable wave number for Pc3-4 pulsations is used in the model, the predicted amplitudes agree with observations.

We next consider the phase relationship between Doppler velocity oscillations and geomagnetic pulsations. The phase differences between Doppler velocity oscillations and geomagnetic pulsations predicted by the model are very sensitive to changes in the geophysical parameters. Consequently, the predicted phase differences are extremely variable, thus making it difficult to specify any definitive phase relationship. The expectation that the phase differences between $V^{*}$ and geomagnetic pulsations should be extremely variable is confirmed by observations. In his statistical study of SIs and Pi2s, Watermann (1987) found that the phase differences are nearly randomly distributed. Jarvis and Gough (1988) and Tedd et al. (1989) studied pulsations in the Pc3-4 range. The former authors found the phase difference between the two data sets at various frequencies is not consistent but changes with time. The latter group found that the phase relationship between the ionospheric and magnetic pulsation signals is highly variable.

The only reasonably definitive statement that can be made about phase is that for a particular set of geophysical parameters the rate of change of Doppler velocity phase with reflection level is relatively gradual for F-region reflections. This is seen in the right-hand parts of Figs. 8 and 9. Note that although rapid phase changes are predicted close to the ionospheric cusps, reflections from these regions, as previously pointed out, are not likely to be observed in practice. Observational results which support this model prediction are presented by Tedd et al. (1989) in their Fig. 7. Menk (1992) also concluded that the Doppler oscillations are essentially in phase at different heights.

Liu (1991) and Liu et al. (1993) commenced with expressions derived by Poole and Sutcliffe (1987) and Poole et al. (1988) and followed analytical methods rather than numerical methods to derive the phase relationships between $b_{x}$ at ground level and $V_{2}$ and $V_{3}$ in the ionosphere. For example, Liu (1991) showed that $V_{2}$ and $V_{3}$ respectively lag and lead $b_{x}$ by $90^{\circ}$. The results in Figs. 8 and 9 show that the phase differences predicted by the numerically evaluated model tend toward these idealized values, particularly in the upper F-region. The analytical derivation provides some insight to the physical mechanisms involved; however, due to their highly idealized nature, they are of questionable practical value. Both observations and numerical modelling demonstrate that the observed phase differences depend on a variety of geophysical parameters. For example, the observations of Watermann (1987) and numerical modelling of Sutcliffe and Poole (1990) show that the phase differences are a function of ULF pulsation frequency. Liu et al. (1993) attempted to use their analytically derived phase differences to establish the causal mechanism relating geomagnetic pulsations and HF Doppler oscillations. However, their observed phase differences for a single event ranged in value from $15^{\circ}$ to $77^{\circ}$ and they concluded that 
the failure to observe values of $\pm 90^{\circ}$ was caused by more than one mechanism contributing to the observations. We thus reiterate the findings of Sutcliffe and Poole (1990) that not only do a combination of mechanisms contribute to the relationships observed between geomagnetic pulsations and HF Doppler oscillations, but that changes in geophysical parameters also alter the relationships for each individual mechanism.

\subsection{Shortcomings and limitations of model}

The model implicitly assumes a one-to-one relationship between Doppler velocity and geomagnetic pulsations; consequently, the model predicts a one-to-one relationship between the respective amplitudes of oscillation. However, observations clearly indicate that this is not generally the situation. Jarvis and Gough (1988) found that the magnetometer and Doppler velocity data do not always exhibit oscillations at the same frequencies at the same time and that they may oscillate at different frequencies. Tedd et al. (1989) presented examples where good correlation is evident between the oscillations of the magnetic field and ionospheric Doppler shift. Notwithstanding this, however, they found that more typical of their data set was poor, or even absence of, activity-wise correlation. The ISP model is not able to, nor intended to, account for these discrepancies. The purpose of the ISP model is to quantify the relationship between geomagnetic pulsations and the ionospheric signatures at times when they are well correlated. A number of causes for poor or lack of correlation can be listed (Tedd et al., 1989; Menk, 1992). RF wave interference effects due to ionospheric irregularities significantly degrade the correlation between geomagnetic pulsations and ionospheric Doppler oscillations. Interference of rays arizing from multipath propagation or from different modes can also produce oscillations of ionospheric parameters in the ULF frequency range, but which are unrelated to geomagnetic pulsations. Jarvis and Gough (1988) and Menk (1992) emphasize that another important cause for poor correlation is the fact that the observations of ionospheric signatures are from a very localised region of the ionosphere, whereas the observations by ground based magnetometers are the integration of oscillating electric fields over a much larger region of the ionosphere.

Menk (1992) points out that instances where observations and model predictions do not agree may indicate where initial assumptions are being violated. The $V^{*}$ height profiles presented in Figs. 8 and 9 and the ISP model predictions presented by Sutcliffe and Poole (1990) are for ionospheric signatures associated with transverse Alfvén waves. Thus disagreement may arise in instances where geomagnetic pulsations are due to cavity mode resonances, fast mode propagating waves, or coupled wave modes. The assumption of transverse Alfvén waves above was to fulfil the requirement for electric and magnetic field profiles through the ionosphere in order to numerically evaluate Eqs. (7) to (10). However, these equations could equally well be evaluated using electric and magnetic field profiles for other wave modes; this was in fact done by Sutcliffe (1993) where the fields for a fast magnetosonic wave propagating in the ionospheric duct (Fujita, 1987) were used to simulate conditions applicable to right-hand elliptically polarized Pc1 pulsations distant from the Pc1 source region.

\section{Conclusion}

In this paper we have reviewed a model which quantitatively accounts for the relationship between geomagnetic pulsations and oscillating frequency shifts in HF radio waves propagating through the ionosphere. We demonstrated how the model can be used to predict the relationship between the Doppler velocity and geomagnetic pulsations and shown how the predicted amplitudes and phases of $V^{*}$ vary with changes in geophysical parameters. In particular, the Doppler velocity depends on electron concentration and is typically more than an order of magnitude greater during the night than during the day, greater in the F-region than in the E-region, and greatest close to the ionospheric cusps. These various predictions are in reasonable general agree- 
ment with observations. However, a comparison of observations with model predictions where all parameters are chosen to be as close as practicable to the observed set has not yet been made. Such a comparison would indicate in which respects the model might be improved. Nevertheless, we can mention some desirable improvements. Electric and magnetic field profiles through the ionosphere which are more representative of the geomagnetic pulsation could be used. A computationally more complex but desirable option would be for a Fourier synthesis of results in time and space. However, even without these improvements, we believe the model reviewed here to be the best currently available to quantify the relationship between geomagnetic pulsations and ionospheric Doppler shifts.

\section{REFERENCES}

Al'Perovich, L. S., A. V. Volgin, P. B. Karpov, E. N. Federov, V. A. Pilipenko, and S. N. Pokhil'ko, Registration of MHD waves in the ionosphere and on the ground, Paper 2.05.56 presented at IAGA Scientific Assembly, Exeter, 1989

Aslin, P. M., M. J. Jarvis, and K. Morrison, Ionosonde signatures of Pc1 pulsations, J. Atmos. Terr. Phys., 53, 343-349, 1991.

Bourdillon, A., J. Delloue, and J. Parent, Effects of geomagnetic pulsations on the Doppler shift of HF backscatter radar echoes, Radio Sci., 24, 183-195, 1989.

Chan, K. L., D. P. Kanellakos, and O. G. Villard, Jr., Correlation of short-period fluctuations of the Earth's magnetic field and instantaneous frequency measurements, J. Geophys. Res., 67, 2066-2072, 1962.

Davies, K. and G. K. Hartmann, Short-period fluctuations in total columnar electron content, J. Geophys. Res., 19, 3431-3434, 1976.

Duffus, H. J. and G. M. Boyd, The association between ULF geomagnetic fluctuations and Doppler ionospheric observations, J. Atmos. Terr. Phys., 30, 481-496, 1968.

Fujita, S., Duct propagation of a short-period hydromagnetic wave based on the international reference ionosphere model, Planet. Space Sci., 35, 91-103, 1987.

Hughes, W. J. and D. J. Southwood, The screening of micropulsation signals by the atmosphere and ionosphere, J. Geophys. Res., 81, 3234-3240, 1976.

Jacobs, J. A. and T. Watanabe, Doppler frequency changes in radio waves propagating through a moving ionosphere, Radio Sci., 1, 257-264, 1966.

Jarvis, M. J. and H. Gough, Digital ionosonde observations of Pc3-4 pulsations across the plasmapause, Planet. Space Sci., 36, 733-745, 1988.

Klostermeyer, J. and J. Röttger, Simultaneous geomagnetic and ionospheric oscillations caused by hydromagnetic waves, Planet. Space Sci., 24, 1065-1071, 1976.

Kolokolov, L. Ye. and A. V. Marakhovskiy, Quick response of the ionospheric F region to the passage of Pi2-type hydromagnetic pulsations, Geomagn. Aeron., 28, 119-120, 1988.

Liu, J. Y., A note on the phase relationship between ULF geomagnetic pulsations and HF-Doppler oscillations owing to the compressional mechanism, J. Geomag. Geoelectr., 43, 777-781, 1991.

Liu, J. Y., Y. N. Huang, and F. T. Berkey, The phase relationship between ULF geomagnetic pulsations and HF Doppler frequency shift oscillations on March 24, 1991, J. Geomag. Geoelectr., 45, 109-114, 1993.

Menk, F. W., Characterization of ionospheric Doppler oscillations in the Pc3-4 and Pi2 magnetic pulsation frequency range, Planet. Space Sci., 40, 495-507, 1992.

Menk, F. W., K. D. Cole, and J. C. Devlin, Associated geomagnetic and ionospheric variations, Planet. Space Sci., 31, 569-572, 1983.

Okuzawa, T. and K. Davies, Pulsations in total columnar electron content, J. Geophys. Res., 86, 1355-1363, 1981.

Poole, A. W. V. and P. R. Sutcliffe, Mechanisms for observed total electron content pulsations at mid latitudes, J. Atmos. Terr. Phys., 49, 231-236, 1987.

Poole, A. W. V., P. R. Sutcliffe, and A. D. M. Walker, The relationship between ULF geomagnetic pulsations and ionospheric Doppler oscillations: Derivation of a model, J. Geophys. Res., 93, 14656-14664, 1988.

Rishbeth, H. and O. K. Garriott, Relationship between simultaneous geomagnetic and ionospheric oscillations, Radio Sci. J. Res., 68D, 339-343, 1964.

Sutcliffe, P. R., Amplitude modulation in ionospheric signatures of ULF pulsations, Planet. Space Sci., 40, 965$971,1992$.

Sutcliffe, P. R., Ionospherically reflected radio wave signatures of left- and right-hand polarized Pc1 pulsations, Paper presented in session GAM 3.8 at 7th IAGA Scientific Assembly, Buenos Aires, 1993.

Sutcliffe, P. R. and A. W. V. Poole, Low-latitude Pc 3 pulsations and associated ionospheric oscillations measured by a digital chirp ionosonde, Geophys. Res. Lett., 11, 1172-1175, 1984.

Sutcliffe, P. R. and A. W. V. Poole, Pc3 pulsations and ionospheric Doppler velocity oscillations, Internal Report MAG IR-85/5, Magnetic Observatory, Hermanus, 1985. 
Sutcliffe, P. R. and A. W. V. Poole, Ionospheric Doppler and electron velocities in the presence of ULF waves, $J$. Geophys. Res., 94, 13505-13514, 1989.

Sutcliffe, P. R. and A. W. V. Poole, The relationship between ULF geomagnetic pulsations and ionospheric Doppler oscillations: Model predictions, Planet. Space Sci., 38, 1581-1589, 1990.

Tedd, B. L., K. D. Cole, and P. L. Dyson, The association between ionospheric and geomagnetic pulsations in the Pc3-4 range at mid-latitudes, Planet. Space Sci., 37, 1079-1094, 1989.

Watermann, J., Observation of correlated ULF fluctuations in the geomagnetic field and in the phase path of ionospheric HF soundings, J. Geophys., 61, 39-45, 1987.

Yumoto, K., K. Takahashi, T. Ogawa, and T. Watanabe, Sc- and Si-associated ULF and HF-Doppler oscillations during the great magnetic storm of February 1986, J. Geomag. Geoelectr., 41, 871-878, 1989. 